\title{
Electronic and atomic kinetics in solids irradiated with free-electron lasers or swift-heavy ions
}

\author{
N. Medvedev ${ }^{\mathrm{a}, *}$, A.E. Volkov ${ }^{\mathrm{b}, \mathrm{c}, \mathrm{d}}$, B. Ziaja ${ }^{\mathrm{a}, \mathrm{e}}$ \\ ${ }^{a}$ Center for Free-Electron Laser Science at DESY, Notkestr. 85, 22607 Hamburg, Germany \\ ${ }^{\mathrm{b}}$ FLNR, JINR, Joliot-Curie 6, 141980 Dubna, Russia \\ ' NRC Kurchatov Institute, Kurchatov Sq. 1, 123182 Moscow, Russia \\ ${ }^{\mathrm{d}}$ LPI of the Russian Academy of Sciences, Leninskij prospekt, 53, 119991 Moscow, Russia \\ e Institute of Nuclear Physics, Polish Academy of Sciences, Radzikowskiego 152, Kraków 31-342, Poland
}

\section{A R T I C L E I N F O}

\section{Article history:}

Received 14 May 2015

Received in revised form 2 August 2015

Accepted 20 August 2015

Available online 2 September 2015

\section{Keywords:}

Free-electron laser

Swift-heavy ion

Electron kinetics

Nonthermal melting

Nonequilibrium

\begin{abstract}
A B S T R A C T
In this brief review we discuss the transient processes in solids under irradiation with femtosecond X-ray free-electron-laser (FEL) pulses and swift-heavy ions (SHI). Both kinds of irradiation produce highly excited electrons in a target on extremely short timescales. Transfer of the excess electronic energy into the lattice may lead to observable target modifications such as phase transitions and damage formation. Transient kinetics of material excitation and relaxation under FEL or SHI irradiation are comparatively discussed. The same origin for the electronic and atomic relaxation in both cases is demonstrated. Differences in these kinetics introduced by the geometrical effects ( $\mu \mathrm{m}$-size of a laser spot vs nm-size of an ion track) and initial irradiation (photoabsorption vs an ion impact) are analyzed. The basic mechanisms of electron transport and electron-lattice coupling are addressed. Appropriate models and their limitations are presented. Possibilities of thermal and nonthermal melting of materials under FEL and SHI irradiation are discussed.
\end{abstract}

(c) 2015 Elsevier B.V. All rights reserved.

\section{Introduction}

Systems far from equilibrium are actively investigated during the last decades (see, e.g., [1-7]). Effects detected in highly excited solids pose a number of challenges from both theoretical and experimental viewpoints [1]. For example, excitation by ultrafast energy deposition into the electronic subsystem of a material produces transient nonequilibrium states, which cannot be described by the standard techniques based on macroscopic quantities or local equilibrium conceptions [8-12].

Extreme initial excitation of the electronic subsystem of a solid is commonly produced in experiments by two ways.

Intense free-electron lasers, the 4-th generation light sources such as FLASH [13], LCLS [14], SACLA [15], FERMI [16], European XFEL [17], produce brilliant extreme ultraviolet and X-ray radiation with photon energies up to $\sim 24 \mathrm{keV}$ [17]. FELs pulse duration reaches a femtosecond timescale [14]. Their intensities are sufficient to trigger material modifications by a single shot. One of the most important advantages of femtosecond FELs is that the pulse duration is comparable with characteristic timescales of

\footnotetext{
* Corresponding author.

E-mail address: nikita.medvedev@desy.de (N. Medvedev).
}

the basic processes in solids, e.g. the typical timescales of nonequilibrium electron cascades in material. A spot of X-ray FEL has a typical size of a $\mu \mathrm{m}$. The photon attenuation length depends on the photon energy, and can be as short as a few tens of nanometers (for VUV at energy around the plasmon minimum), or as long as microns (for hard X-rays) $[18,19]$. Thus, material modifications produced by X-ray FEL are typically of a micron size.

Swift heavy ions (SHI, $M \geqslant 20 m_{\mathrm{p}}, m_{\mathrm{p}}$ is the proton mass, $E>1 \mathrm{MeV} / \mathrm{amu}$ ) decelerated in the electronic stopping regime excite, in the nanometric vicinities of their trajectories, primary electrons (so-called delta-electrons) up to energies similar to those of FEL-irradiation. For instance, UNILAC accelerator at GSI produces electrons with energies up to $\sim 24 \mathrm{keV}[20,21]$ by an impact of a nonrelativistic heavy ions with energies around the Bragg-peak realizing the electronic stopping [22]. SHI impacts can stimulate structure and phase transformations forming nanometric latent tracks along the SHI trajectories in a bulk. Their length can be of a few tens of microns or even longer, depending on the SHI energy and stopping power [22]. Surface damage in a shape of craters [23] and/or hillocks [24,25] can also be formed during an SHI irradiation.

In both cases, FEL and SHI irradiation, initially excited electrons scatter further elastically (exchanging kinetic energy with the 
atoms without secondary electron excitation) and inelastically (impact ionization of secondary electrons), ultimately relaxing to the low-energy states of the conduction band. Auger-decay of core holes brings them up to the valence band via a sequential cascade of events. Excess energy is later transferred to lattice atoms. These relaxation processes in the electron subsystem are physically the same for FEL or SHI irradiation. The differences are only introduced by two effects: (a) initial spectra of excited electrons that are different after photoabsorption and after an SHI impact; (b) geometry of the problem is different due to the difference in the spatial scales mentioned above. We will discuss these similarities and differences in the next sections.

The similarity of the physical processes during relaxation leads to mutual benefits for the communities investigating FEL and SHI irradiation. For example, pump-probe experiments studying transient electron kinetics are much easier to perform with FELs, since advanced synchronization schemes can achieve a femtosecond resolution [26,27]. The data on electron cascading in solids can then be utilized for modeling of SHI irradiation, which can be very useful considering that femtosecond resolution has not yet been achieved in SHI experiments.

A number of subsequent stages of nonequilibrium appear after ultrafast high-energy deposition into the electronic subsystem of a material:

(i) Initially excited electrons (on the femtosecond timescale) retain nonthermalized distribution until their thermalization (which may take up to a few hundred femtoseconds $[8,11])$. During first ten femtoseconds [21,28,29], they are accompanied by the Auger cascades of core-holes, also being out of ionization equilibrium.

(ii) On the sub-picosecond timescale [9], the distribution of atomic energies in a solid under intense excitation may as well be nonthermal (non-Maxwellian).

(iii) Later, the electron ensemble may thermalize at temperatures considerably higher than the lattice temperature. Electron-lattice coupling equilibrate these temperatures during a few to a few tens of picoseconds [1,2,8,30,31].

(iv) Additionally, effects of extensive spatial temperature and carrier density gradients can be important for the relaxation kinetics of the localized initial excitation, governing particle and energy transport [21,32-34].

Further material damage may proceed via a number of different channels: (a) thermal lattice heating by electron-phonon (or, more generally, electron-lattice) coupling; (b) nonthermal melting via modification of the interatomic potential caused by high excitation of the electron subsystem of a solid; (c) Coulomb explosion due to space-charge distribution resulting from transient spatial separation of excited electrons and lattice ions; (d) accumulation of structure defects during relaxation of electronic excitations, e.g. creation of color centers in alkali-halides due to creation and decay of self-trapping excitons. The contribution of these mechanisms depends on the peculiarities of the irradiation and target parameters.

In this paper we will only address the first two channels which realize in all targets, while the latter two are excluded from current considerations for the following reasons.

The channel " $d$ " for electronic excitation is realized only for a number of wide band gap dielectrics (see e.g. [35,36] where the mechanism of point defect appearing due to creation and decay of self-trapped excitons is discussed). Also, multiple laser shots or prolonged radiation exposures are necessary for accumulation of point defect concentrations necessary for stimulation of lattice instability. Such multi-shot effects will not be discussed in this paper.
The Coulomb explosion (channel ' $c$ ') plays an important role only for finite systems such as molecules and nanoclusters: e.g. it was shown in Refs. [37,38] that increase of the size of a nanocluster results in decrease of the effect of Coulomb explosion for the interior of the cluster. The explosion then mostly affects its outer layer. Extrapolating, one can assume that in a bulk material Coulomb explosion does not occur, except for the near-surface region where the emitted electrons could create long-lasting charge non-neutrality. In this paper we will not consider such near-surface effects, focusing on the material deeply inside the bulk, far from a surface.

\section{Electron kinetics}

At present, it is hardly possible to solve many-body timedependent Schrödinger equation to trace the evolution of the highly-excited electron ensemble in a solid on the $a b$ initio level. Thus, for a calculation of nonequilibrium electron kinetics different approximations are used. In particular, one of the most popular is the one-particle semi-classical approximation resulting in Boltzmann kinetic equation for electrons [4,8,38-42]. Event-by-event Monte Carlo (MC) models $[34,43,44]$ of independent electron scattering can be essentially considered as belonging to the same class of methods: introduction of correlations into the electron ensemble within the MC scheme (such as done e.g. in $[11,45]$ ) allows to include electron-electron interaction and Pauli's blocking effects similarly to the Boltzmann collision integral for electrons in solids.

The difference in the initial excitation of the electron subsystem by FEL or SHI appears, first of all, because photoabsorption is realized by quanta, whereas an ion impact excites the whole spectrum of electrons. However, the subsequent relaxation processes are similar. For example, in Fig. 1 the evolution of the total electron density is shown in both irradiation cases. Fig. 1 shows evolution of the electron density in diamond irradiated with $1 \mathrm{fs}$ FEL pulse, photon energy of $24 \mathrm{keV}$, fluence of $1 \mathrm{~mJ} / \mathrm{cm}^{2}$, vs irradiation with Ca ion with the energy of $457 \mathrm{MeV}$ (11.4 MeV/amu). This particular ion energy is chosen for a better comparison of the two scenarios, because the maximum electron energy provided in the ion collision is $\sim 24 \mathrm{keV}$ i.e. the same as in the FEL case discussed.

Differing timescales of the electron cascading can be observed before the saturation of the densities follows. The electron cascading started by photoabsorption at the energies around $24 \mathrm{keV}$ excited with an FEL pulse takes around $\sim 120 \mathrm{fs}$. After that, the excited electrons do not have sufficiently high energies to produce impact ionization, as will be discussed in the next section. In contrast, after the SHI impact, there is a lot of low-energy electrons in the first generation ionized by the ion which relax much faster (95\% of electrons are produced already by $40 \mathrm{fs,} 99 \%$ are excited by $90 \mathrm{fs}$ ). The relaxation of a minority of high-energy electrons still lasts a time comparable to that produced by an FEL pulse ( $\sim 90 \mathrm{fs,}$ as will become clear in the Section 2.2); these very few electrons, however, are highly energetic, thus, may be important to consider.

To emphasize the similarities and differences, below we will discuss the two cases in more detail.

\subsection{FEL irradiation}

Owing to a typical micron-size of a laser spot, homogeneous excitation can usually be assumed and periodic boundary conditions can be used for modeling. That significantly simplifies theoretical description, since zero-dimensional models could then be applied, tracing only evolution of the electron distribution in the energy space. XCASCADE ${ }^{1}$ code [46], and its predecessors, were used

\footnotetext{
${ }^{1}$ N. Medvedev and B. Ziaja. XCASCADE, a Monte Carlo tool for modeling ultrafast electron cascading in solids after X-ray irradiation. DESY, Hamburg, 2014.
} 


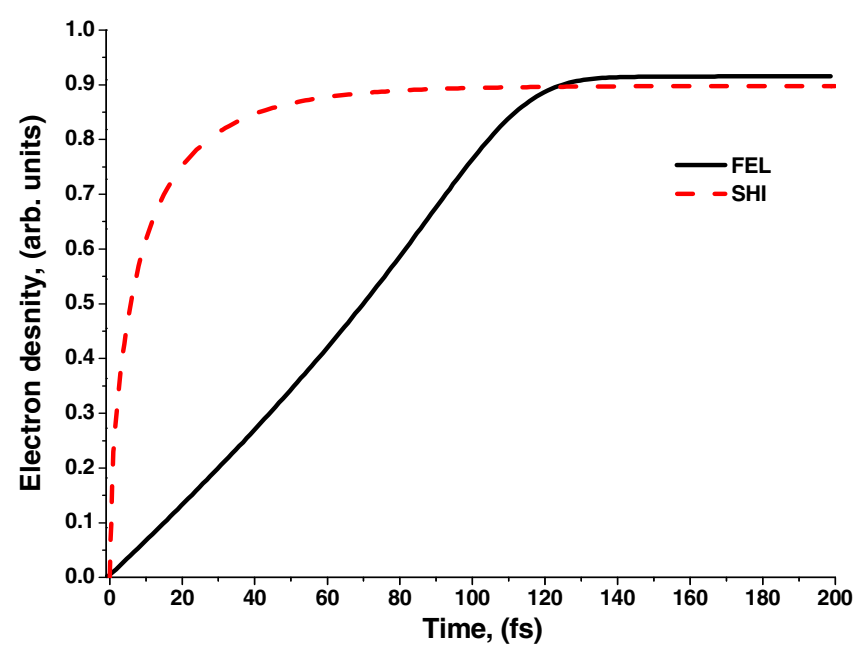

Fig. 1. Evolution of the electron density in diamond irradiated with: an FEL pulse of $1 \mathrm{fs}$, photon energy $24 \mathrm{keV}$, fluence of $1 \mathrm{~mJ} / \mathrm{cm}^{2}$ (FEL), or Ca ion with energy 11.4 MeV/amu (SHI).

to study evolution of electron distribution in different materials irradiated with an FEL pulse $[11,45,47]$. This code is also used to obtain the data presented in Fig. 1, and the results discussed below.

Fig. 2 shows the transient electron distribution in diamond after irradiation with an FEL pulse (the same parameters as for Fig. 1). The initial electron distribution is formed by electrons excited from the valence and conduction bands or from deep shells of carbon atoms, shifted to high energies by the amount of the photon energy $(\sim 24 \mathrm{keV})[45]$. The secondary peak around $300 \mathrm{eV}$ is formed by Auger-electrons appearing due to decays of K-shell holes of carbon atoms which occur on a characteristic time of $\sim 8$ fs [48]. Relaxation of the high-energy nonequilibrium tail of the distribution is predominantly governed by the impact ionizations of secondary electrons during development of electron cascades. Duration of these cascades depends on the initial excitation level (photon energy and fluence), and the material parameters [46,47]. For the initial electron energy of $\sim 24 \mathrm{keV}$ in diamond, these cascades last $\sim 120$ femtoseconds [46], as we also have seen in Fig. 1.

Low-energy electrons are usually treated as non-interacting electron gas. More detailed studies, which included interactions between excited electrons, demonstrated partial thermalization of electrons with energies below $\sim 10 \mathrm{eV}$ occurring within a few femtoseconds $[10,11,49]$. This low-energy fraction of electrons follows then the Fermi-Dirac distribution with still increasing temperature being provided from interaction with high-energy nonequilibrium electrons $[11,50]$.

Detailed analysis demonstrated that the transient nonequilibrium shape of the electron distribution in different materials (metals [11], semiconductors [45], insulators [47]) during and after an FEL pulse follows the so-called 'bump-on-hot-tail' distribution [51], similar to that shown in Fig. 2. It consists of a large number of electrons populating low-energy states with (nearly) thermal distribution, while a minority of electrons remains in the long nonequilibrium tail of the distribution at high energies. Although the number of such high-energy electrons is small compared to those within the low-energy thermalized electron fraction, they possess rather large amount of energy, and therefore cannot be neglected and assumed to be thermalized at femtosecond timescales [11]. For highly excited warm-dense matter and plasma, this shape of the transient electron distribution was also confirmed by different methods: e.g. solutions of Boltzmann kinetic equation [10], and molecular dynamics simulations [49].

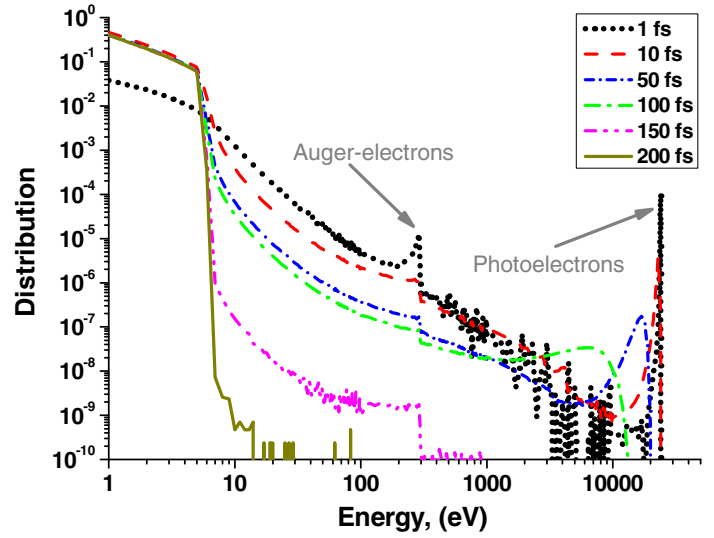

Fig. 2. Evolution of the electron distribution function in diamond irradiated with an FEL pulse of $1 \mathrm{fs}$, photon energy $24 \mathrm{keV}$, fluence of $1 \mathrm{~mJ} / \mathrm{cm}^{2}$.

As it was shown in Ref. [11], different experimental techniques have access to different parts of the electron distribution function on femtosecond timescales. Thus, the 'bump-on-hot-tail' shape of transient nonequilibrium electron distribution must be taken into account for interpretation of experimental data [11,51]. For example, interpretations based on the assumption of thermal equilibrium produced contradicting results: a spectrum from radiative decay of L-shell holes in FEL-irradiated aluminum showed electron temperatures of $\sim 1 \mathrm{eV}$ [52], whereas Bremsstrahlung spectra from the same system were attributed to the electron temperatures of $\sim 40 \mathrm{eV}$ [53]. But such results could be explained by the fact that in the radiative decays L-shell holes are filled by electrons from the thermalized low-energy part of the transient electron distribution, while Bremsstrahlung spectra were formed by high-energy nonequilibrium tail of the 'bump-on-hot-tail' distribution [11].

\subsection{SHI impact}

The developed Monte Carlo code TREKIS ${ }^{2}$ [54] was used for modeling of electron kinetics in different materials after a swiftheavy ion impact (shown in Fig. 1 above, and those discussed below). Transient electron distribution after an SHI impact demonstrates qualitatively similar behavior to the FEL-case $[21,55]$. Fig. 3 presents the evolution of electron distribution in diamond after irradiation with a Ca ion with the energy of $11.4 \mathrm{keV} / \mathrm{amu}$. Its initial shape is different from FEL case, because an ion excites electrons of different energies. Their spectrum scales approximately as $\sim 1 / E^{2}$, except for low energies where it exhibits a plasmon peak [56].

This spectrum then quickly relaxes to the shape similar to the 'bump-on-hot-tail' distribution discussed above. However, since: (i) in the initial electron spectrum from SHI impact there is no peak similar to that of photoelectrons as after an FEL pulse, and (ii) a majority of primary electrons occupies low energy states, it makes the relaxation of the initial electron spectra somewhat faster in SHI-irradiation case (Fig. 3 vs Fig. 2). By the time of $\sim 90 \mathrm{fs}$ most of the electrons are already in the low-energy states (99\% of them are below the ionization threshold), however, a minor amount of electrons up to $\sim 30 \mathrm{eV}$ is still present. Their number is so small that the secondary impact ionizations produced by them are almost not noticeable compared with the number of already excited electrons (the saturation regime in Fig. 1).

However, taking into account possible effects of the nonequilibrium electron distribution is important because it may affect inter-

\footnotetext{
${ }^{2}$ N. Medvedev, R. Rymzhanov and A.E. Volkov, TREKIS: a Monte Carlo tool for modeling Time-Resolved Electron Kinetics in SHI Irradiated Solids. 2014.
} 


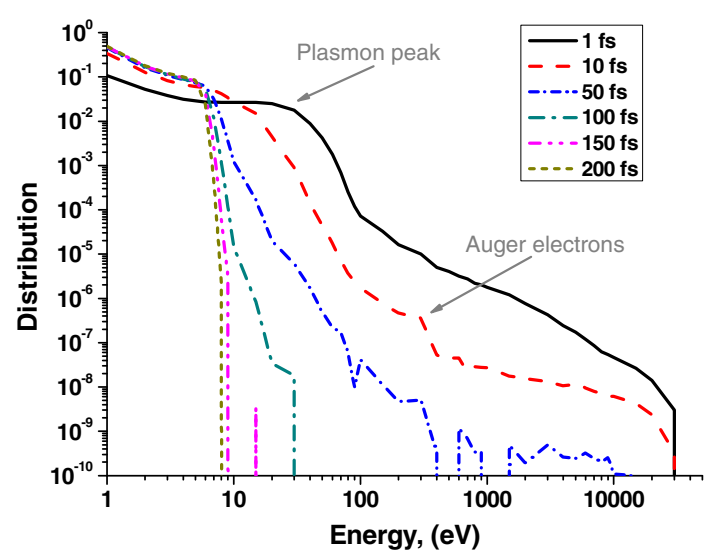

Fig. 3. Evolution of the electron distribution function in diamond irradiated with $\mathrm{Ca}$ ion (457 MeV, or $11.4 \mathrm{MeV} / \mathrm{amu}$ ).

pretation of experiments: femtosecond diagnostics ( $<100 \mathrm{fs}$ ) based on radiative photoemission or on Auger-electrons spectra [57], must keep in mind that they have access only to the low-energy part of the nonequilibrium distribution, and therefore estimated temperatures do not completely describe the excited electron ensemble. The shorter timescales a diagnostic tool probes, the more pronounced are nonequilibrium effects.

Important difference between FEL and SHI irradiation occurs in the spatial distribution of the initial electronic excitations. In case of an SHI impact, homogeneous excitation cannot be assumed, i.e. periodic boundary conditions only along the ion-trajectory can be implemented in the cylindrical geometry $[33,58]$. Strongly localized initial excitation spreads out radially on femtosecond timescales, significantly changing radial profile of the electron density (and energy), as shown in Fig. 4. Initial ballistic transport of electrons gradually turns into diffusive propagation on the scales of a hundred femtosecond, alongside with the energy distribution relaxation [55,59]. But again, nonequilibrium part of fast electrons is important, since they possess a large amount of energy, and are spreading ballistically on femtosecond timescale, in contrast to diffusive motion of low-energy electrons [21,59].

The amount of energy kept by electrons with energies above a certain value is shown in Fig. 5 as a function of time. We can see that at the beginning about $95 \%$ of the energy provided by the SHI is accumulated in the electrons with energies above $10 \mathrm{eV}$, which are capable of performing secondary impact ionizations. Electrons with energies above $100 \mathrm{eV}$ possess over $40 \%$ of the energy forming the ballistic front shown in Fig. 4. Due to the secondary cascading, electrons lose their energy and the fraction of high-energy electrons quickly decreases. By the time of $\sim 90 \mathrm{fs}$, less than $1 \%$ of the energy is kept by the electrons with energies above $10 \mathrm{eV}$. This indicates that most of the electrons are already slow, as we could also see in the electron spectrum, Fig. 3. Slow electrons exhibit diffusive behavior instead of the ballistic one [21,59], implying that in case of diamond irradiated with an SHI of 11.4 MeV/amu, after the time of $\sim 90$ fs a thermodynamic approach can be used for description of the excited electron ensemble.

It is also important to mention that many MC models are using a hard cut-off energy, stopping electrons as soon as their energy drops below a certain value (typically, around $10 \mathrm{eV}$ ). Out of this condition, the so-called total deposited dose vs radius is determined [32-34]. Obtained radial profile of the deposited dose is often used as an initial condition for further thermodynamic calculations, such as two-temperature model (see e.g. [60]). Such an approach, however, is inherently inconsistent, since neither the particle, nor the energy diffusion for low-energy electrons actually stops below any cut-off energy, therefore the spatial and temporal

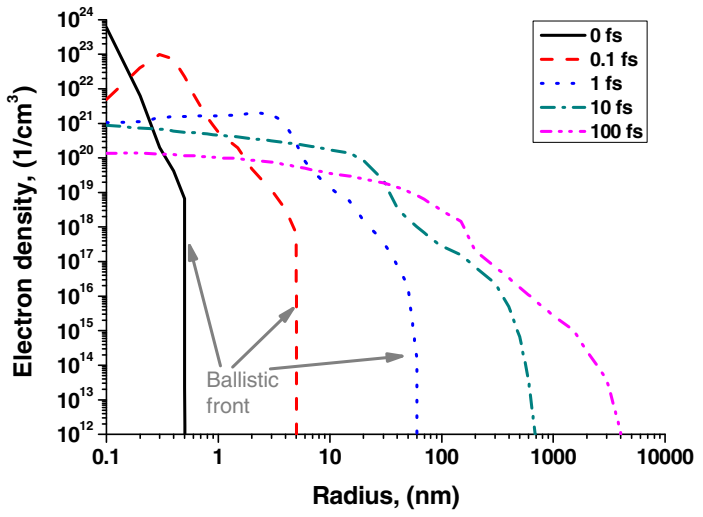

Fig. 4. Radial electron distribution in diamond irradiated with $\mathrm{Ca}$ ion (457 MeV, or 11.4 MeV/amu).

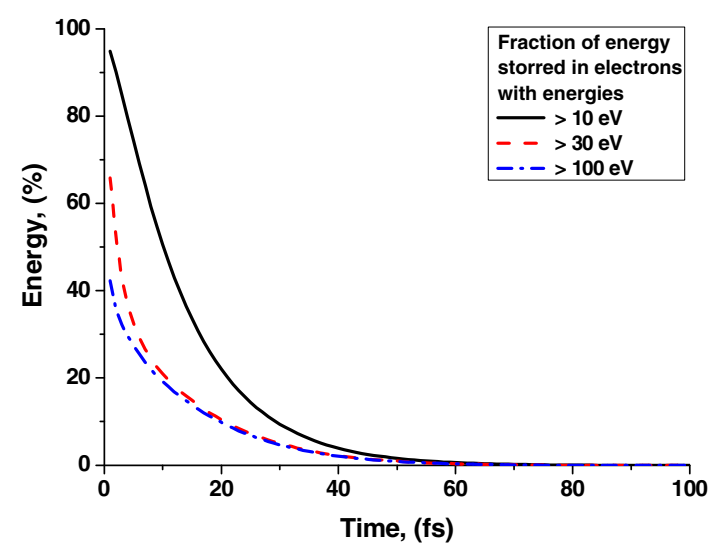

Fig. 5. Fraction of energy possessed by the electrons with energies above a certain value in diamond irradiated with $\mathrm{Ca}$ ion (457 MeV, or $11.4 \mathrm{MeV} / \mathrm{amu})$.

electron density evolution cannot be decoupled. This implies that the 'total dose' calculated in this manner does not correspond to any particular time instance, and, therefore, should not be used as the initial condition for further quantitative modeling [61]. An appropriate way to use the data characterizing the excited electron ensemble must: (a) account for the instance of electron thermalization, after which thermodynamic methods can be used, and (b) use transient electrons and energy distributions at a chosen time, without introducing artificial energy cut-offs [59,62].

\section{Atomic dynamics stimulated by electron-lattice coupling}

As we have seen in the previous section, within $\sim 100 \mathrm{fs}$ after FEL or SHI irradiation, highly excited electrons are relaxing into low-energy thermalized states. During and after this relaxation, these electrons are coupling to the lattice, which may stimulate structure transformations in a material. Electron-lattice coupling is dominated by two effects following from the transfer of kinetic and potential energies to atoms. The direct increase of the kinetic energy (temperature) of the lattice atoms is often referred to as the 'electron-phonon coupling' in the literature [63]. The change of the potential energy modifies the interatomic potential that may cause the so-called 'nonthermal melting' of lattice $[64,65]$. Below we will discuss these effects as well as the models and approximations with which they can be addressed. After that, we will give a few examples of atomic kinetics where the both effects and their interplay are investigated for conditions typical for SHI and FEL irradiation. 


\subsection{Thermal electron-lattice coupling}

Before we present the models applicable to describe femtosecond electron-lattice energy exchange, let us start with the consideration of an often-used approximation for description of electronlattice kinetic energy exchange: the phonon approximation for the atomic dynamics. In addition to splitting the atomic Hamiltonian into two parts describing the equilibrium atomic positions and their displacements (see discussion on Eq. (2) below), phonon approximation also assumes that [63]: (a) atomic motion can be well approximated by harmonic oscillations (which means that atoms are located close to the minimum of their potential); (b) a perfect periodicity in the atomic lattice allows to decouple such harmonic oscillators; (c) all atoms interact with their neighbors at least within their correlation length (phonon wave-length), so that their motion can be described in terms of collective variables [66]. These assumptions are usually satisfied for conditions realized for pico- and nano-second laser pulses. However, strictly speaking, after a femtosecond laser pulse irradiation, or an SHI impact, none of the approximations $(\mathrm{a}-\mathrm{c})$ is valid: the lattice can be heated to a high temperature or even nonthermally melted, thus, the interatomic potential is strongly unharmonic and quickly changing in time; they can be locally excited and 'melted' on a scale smaller than a phonon wave-length (especially in case of an SHI impact), which breaks the periodicity; interactions of atoms require times longer than the distance between them divided by the speed of sound - that makes a characteristic timescale of collective motion to be at least on the order of a few tens of femtoseconds (inverse phonon frequency). This is much longer than the femtosecond scales of processes considered for a femtosecond laser or SHI irradiation. Therefore, more general methods must be used to describe electron-lattice coupling on femtosecond time scales $[31,66]$.

There are two standard approaches for describing interaction of the electrons with lattice in solids (and dense plasmas) beyond the phonon approximation: (i) free-electron approximation, coming from the plasma physics, and (ii) tight-binding approximation, borrowed from chemistry.

(i) The free-electron approximation assumes weak interaction between electrons and ions, when a gas of free electrons is scattering on a dynamically-coupled atomic ensemble. This allows to solve equations of motion for electrons and atoms independently accounting only for a minor corrections coming from their scattering events. This approximation results in Boltzmann kinetic equation for electrons where the electron-lattice interaction is included via electron-ion collision integral. The collision integral describing the kinetic energy transfer to the atomic subsystem depends on the full potential of electron-ion interaction, affected by the atom-atom dynamical and spatial correlations [67]:

$$
\begin{aligned}
I_{e-a t}^{\text {free }}= & -\frac{4}{(2 \pi)^{5} \hbar^{2}} \int d \mathbf{k}_{i} d \mathbf{k}_{f}|V(\mathbf{q})|^{2}\left\{f_{\mathbf{k}_{f}}(1\right. \\
& \left.\left.-f_{\mathbf{k}_{i}}\right) S(-\mathbf{q},-\omega)-f_{\mathbf{k}_{i}}\left(1-f_{\mathbf{k}_{f}}\right) S(\mathbf{q}, \omega)\right\},
\end{aligned}
$$

where $E_{\mathbf{k}}=\left(\hbar^{2} \mathbf{k}^{2}\right) / 2 m_{e}$ is the kinetic energy of a free electron; $\mathbf{q}=\mathbf{k}_{f}-\mathbf{k}_{i}$ is the vector of momentum transferred by an electron during scattering from the initial $\left(\mathbf{k}_{i}\right)$ to the final $\left(\mathbf{k}_{f}\right)$ state; $V(\mathbf{q})$ is a Fourier-transform of the full electron-ion interaction potential; $f_{k}$ is the distribution function of the free-electron gas; and $S(\mathbf{q}, \omega)$ is the dynamic-structure factor (DSF) which is a Fourier-transform of the pair correlation function of the atomic ensemble [66].

The DSF formalism is actively employed for describing neutron scattering on solids [63,66,67], X-ray scattering [68-
70], and electrons in liquid and amorphous materials [7173] and metals [31,74]. For structured solids, it is often rewritten in terms of the complex-dielectric function [75] utilizing fluctuation-dissipation theorem [76].

(ii) In contrast, the tight-binding approximation assumes that electrons are closely following the atomic motion, which allows to split the atomic Hamiltonian into two parts [63]:

$$
H_{a t}\left(\left\{\mathbf{R}_{i}(t)\right\}\right)=H\left(\left\{\mathbf{R}_{0}\right\}\right)+\delta H\left(\left\{\delta \mathbf{R}_{i}(t)\right\}\right),
$$

where the equilibrium part of the atomic Hamiltonian $H\left(\left\{\mathbf{R}_{0}\right\}\right)$ produces the equilibrium band-structure for electrons. After that, the electrons are assumed to be quasi-particles populating this band-structure (instead of a free-electron-gas dispersion curve). Interaction of such electrons with lattice realizes only via their interaction with atomic displacements $\delta \mathbf{R}_{i}$ from their equilibrium positions: $\delta H\left(\left\{\delta \mathbf{R}_{i}\right\}\right)$.

Note that instead of the equilibrium atomic positions forming the lattice structure as assumed in the phonon approximation discussed above, one can trace evolution of the atomic positions stepby-step replacing $H\left(\left\{\mathbf{R}_{0}\right\}\right)$ by $H(\{\mathbf{R}(t-\delta t)\})$.

It is worth to note that neglecting this so-called nonadiabatic interaction corresponds to the Born-Oppenheimer approximation for the electrons: electrons are then instantly readjusting on the new molecular orbitals that are evolving in time due to the atomic motion. This approximation is often used in $a b$ initio molecular dynamics codes such as DFT-MD. As was discussed in Ref. [30], Born-Oppenheimer approximation neglects electron-phonon (electron-ion) coupling, which can be described only beyond adiabatic effects.

Now, only the displacement part of the Hamiltonian enters the electron-lattice scattering within the tight-binding approximation instead of the full potential; the remaining part is 'compensated' by substituting electrons by quasi-particles on the new dispersion curves of the band-structure. Within this approximation, the electron-ion collision integral can be written as (see details in [30]):

$$
\begin{aligned}
I_{e-a t}^{T B}= & \frac{2 \pi}{h} \sum_{f=1}^{N}\left|M_{e-a t}\left(E_{i}, E_{f}\right)\right|^{2} \\
& \times\left\{\begin{array}{l}
f_{e}\left(E_{i}\right)\left[2-f_{e}\left(E_{f}\right)\right]-f_{e}\left(E_{f}\right)\left[2-f_{e}\left(E_{i}\right)\right] g_{a t}\left(E_{i}-E_{f}\right), \text { for } i>f \\
f_{e}\left(E_{i}\right)\left[2-f_{e}\left(E_{f}\right)\right] g_{a t}\left(E_{i}-E_{f}\right)-f_{e}\left(E_{f}\right)\left[2-f_{e}\left(E_{i}\right)\right], \text { for } i<f
\end{array}\right.
\end{aligned}
$$

where electron distributions are now rewritten in the energy space instead of the momentum one (cf. Eq. (1)), populating the energy levels $E_{i}$ and $E_{f}$ (the eigenstates of the transient Hamiltonian $H(\{\mathbf{R}(t)\})) ; g_{a t}(E)$ is the integrated atomic distribution (the integral of the Maxwellian distribution with a transient ion temperature), and $M_{e-a t}\left(E_{i}, E_{f}\right)$ is the matrix element describing the scattering of an electron on the atomic displacement during the time-step $\delta t$, $\delta H\left(\left\{\delta \mathbf{R}_{i}(\delta t)\right\}\right)[30]:$

$$
M_{e-a t}\left(E_{i}, E_{f}\right)=\frac{1}{2}(\langle i(t-\delta t) \mid f(t)\rangle-\langle f(t-\delta t) \mid i(t)\rangle)\left(E_{j}-E_{i}\right) .
$$

The matrix element depends on the overlap of the electronic wave functions (the eigenfunctions of the Hamiltonian) on the previous $(|i(t-\delta t)\rangle$ and $|f(t-\delta t)\rangle)$ and the current time steps $(|i(t)\rangle$ and $|f(t)\rangle)$. This reflects the fact that the matrix element describes the electron transitions between the levels induced by the atomic displacements. In this formalism there is no distinction between the valence and the conduction band electrons, and all the energy levels (molecular orbitals) are addressed in the same unified manner. 


\subsection{Nonthermal melting}

Nonthermal melting naturally occurs in any ab initio molecular dynamics (MD) [64,65,77]. It is merely a result of the evolution of the atomic potential energy surface, which can be generally written as:

$\Phi(\{\mathbf{R}(t)\}, t)=\sum_{j=1}^{N} f_{e}\left(E_{j}\right) E_{j}(t)+E_{\text {rep }}(\{\mathbf{R}(t)\})$,

where the attractive part is formed by the electrons via the electron distribution function $f_{e}\left(E_{j}\right)$ populating the transient energy levels $E_{j}$ (electron band structure) summed up over all these energy levels $N$; $E_{\text {rep }}$ is the core-core repulsive potential of ions.

Since the potential energy surface depends on the transient distribution of electrons, electronic excitation directly affects the interatomic potential $[64,65,77]$. When sufficient number of electrons is excited to antibonding states, the atomic potential is no longer attractive, and atoms start to experience new forces bringing them out of their former equilibrium positions. Note that in contrast to the Coulomb explosion, during the nonthermal melting the charge neutrality is preserved, and only the distribution function of electrons changes.

\subsection{Examples: thermal and nonthermal melting under FEL irradiation}

Below we will consider three examples of material damage under an FEL irradiation: (a) thermal melting of silicon [30]; (b) an interplay of thermal and nonthermal melting in silicon [30]; (c) purely nonthermal solid-to-solid phase transition: ultrafast graphitization of diamond $[50,78,79]$.

As discussed in the previous section, the typical shape of the transient electron distribution function, the 'bump-on-hot-tail', allows simplifying the theoretical description of the nonequilibrium electron ensemble on femtosecond timescales [78]. The majority of low-energy electrons that are in near-thermalequilibrium already at femtosecond time-scale can be described with the Fermi distribution. The minority of high-energy electrons far from equilibrium must be addressed with a nonequilibrium technique; in our case, we use individual particle Monte Carlo method of event-by-event simulations discussed above [30,78]. For tracing the atomic dynamics, we employ molecular dynamics (MD) on the time-dependent potential energy surface obtained from the transferable tight-binding Hamiltonian (see Ref. [78] for all the details), which naturally accounts for nonthermal effects. Nonadiabatic coupling between electrons and ions is additionally introduced within the tight-binding approximation according to Eqs. (3) and (4). This hybrid scheme is incorporated within the recently developed XTANT code [30].

Fig. 6 presents the atomic structure of solid silicon irradiated with an FEL pulse under a normal incidence characterized by $1 \mathrm{keV}$ photon energy, $10 \mathrm{fs}$ duration (full width at half-maximum of the Gaussian temporal profile), and the photon fluence providing the absorbed dose of $0.7 \mathrm{eV} /$ atom. As it was shown in Ref. [30], in this case the atomic dynamics is governed by the transfer of kinetic energy (heating of the lattice described by Eqs. (3) and (4)). This dose is just above the thermal melting of silicon $\left(E_{t h}=0.65 \mathrm{eV} /\right.$ atom). Fig 6. demonstrates that the phase transition to the transient low-density liquid state of silicon [80]. This state is characterized by the melted atomic structure, which has no long order in the atomic positions but with the kept local order (no amorphization) and semi-metallic electronic state (see [30]). Such melting occurs on a scale of a few ps, started by the fast electronic band gap collapse, while it takes a few picoseconds until electron and lattice temperatures fully equilibrate via heating of the atomic system due to nonadiabatic electron-ion coupling [30].
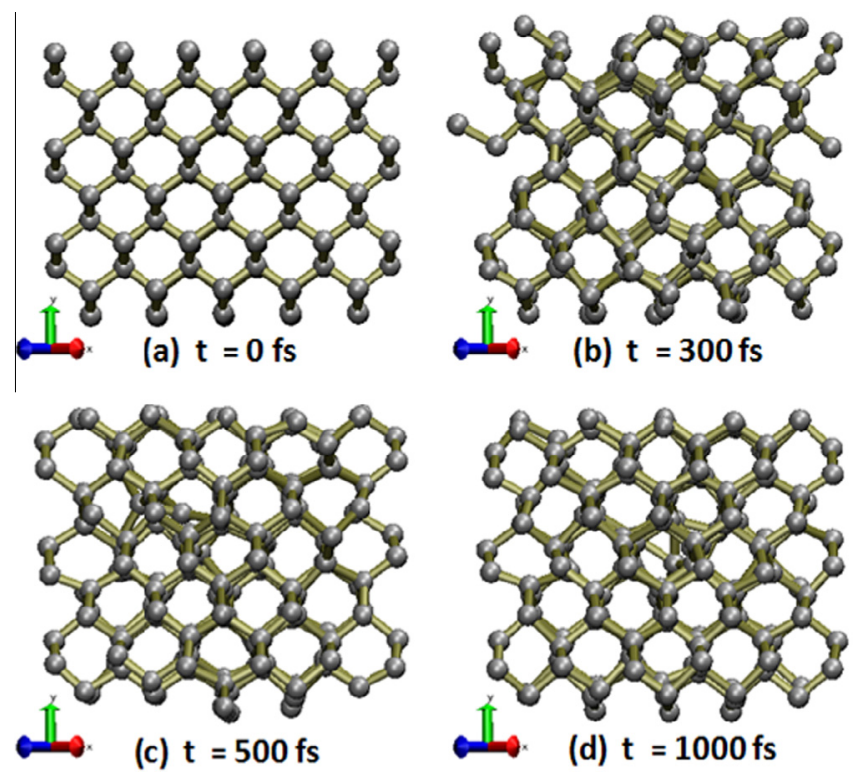

Fig. 6. Atomic snapshots of the thermal melting of silicon under FEL irradiation of $1 \mathrm{keV}$ photon energy, $10 \mathrm{fs}$ pulse, $0.7 \mathrm{eV} /$ atom absorbed dose. The figure is reproduced from Ref. [30].

In contrast, Fig. 7 demonstrates nonthermal melting of silicon, irradiated with an FEL pulse with the higher photon fluence providing the dose of $1 \mathrm{eV} / \mathrm{atom}$ and the same other parameters as in Fig. 6. In this case, the absorbed dose is above the damage threshold leading to amorphization into high-density liquid [80] $\left(E_{t h}=0.9 \mathrm{eV} /\right.$ atom [30]). This amorphization occurs as an interplay between the thermal heating via electron-ion coupling (Eqs. (3) and (4)) and nonthermal melting resulted from modification of the interatomic potential (Eq. (5)). The interatomic potential is softened by the excitation of electrons when more than $4 \%$ of the valence band electrons are promoted to the antibonding states of the conduction band [30]. This results in the so-called 'phonon squeezing', where atoms are trying to adjust to the new potential energy surface formed by excited electrons [81]. Simultaneously, electron-ion coupling provides atoms with sufficient amount of the kinetic energy to overcome the reduced barrier for amorphization [30]. This nonthermal melting is very fast, and amorphization occurs already within $300-500 \mathrm{fs}$ after the exposure to the laser pulse.

Fig. 8 demonstrates an example of a purely nonthermal phase transition: solid-to-solid phase transition from diamond to graphite. Diamond irradiated with FEL providing the dose of $0.85 \mathrm{eV} /$ atom (graphitization threshold is $E_{t h}=0.7 \mathrm{eV} /$ atom [78]) turns into graphite within some $50 \mathrm{fs}$ after the energy is delivered to the low-energy (valence and conductions band) electrons. In the present example of $10 \mathrm{keV}$ photon irradiation, the electron cascade takes around $\sim 100$ fs to bring the energy from high-energy part of the nonequilibrium distribution to the low-energy states of the valence and conduction band, as discussed in the Section 2. After that, the atoms experience a modified potential energy surface, and relax into the new phase of graphite. This happens extremely fast, much faster than nonthermal melting in silicon, because here the lattice is not required to amorphize, but only atoms to shift to the nearest positions corresponding to the new equilibrium solid state. As can be seen in Fig. 8, these are the positions in the graphite planes, while the $\mathrm{sp}^{3}$-hybridization bonds are breaking. Diamond graphitization after exposure to a femtosecond FEL pulse was studied experimentally in Ref. [79]. More related experiments are currently ongoing. 


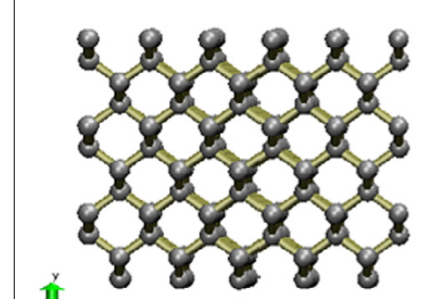

(a) $\mathrm{t}=\mathbf{0}$ fs

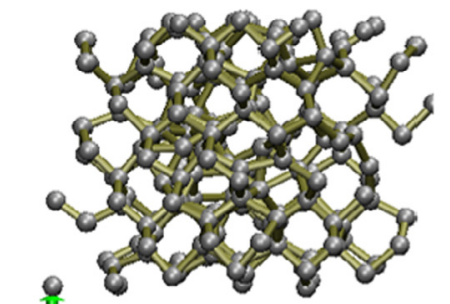

(c) $\mathrm{t}=\mathbf{5 0 0 \mathrm { fs }}$
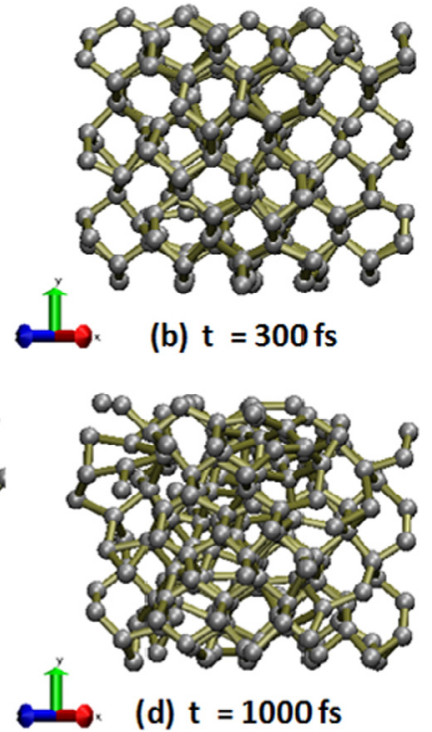

(d) $\mathrm{t}=1000 \mathrm{fs}$
Fig. 7. Atomic snapshots of the nonthermal melting of silicon under FEL irradiation of $1 \mathrm{keV}$ photon energy, $10 \mathrm{fs}$ pulse, $1 \mathrm{eV} /$ atom absorbed dose. The figure is reproduced from Ref. [30].
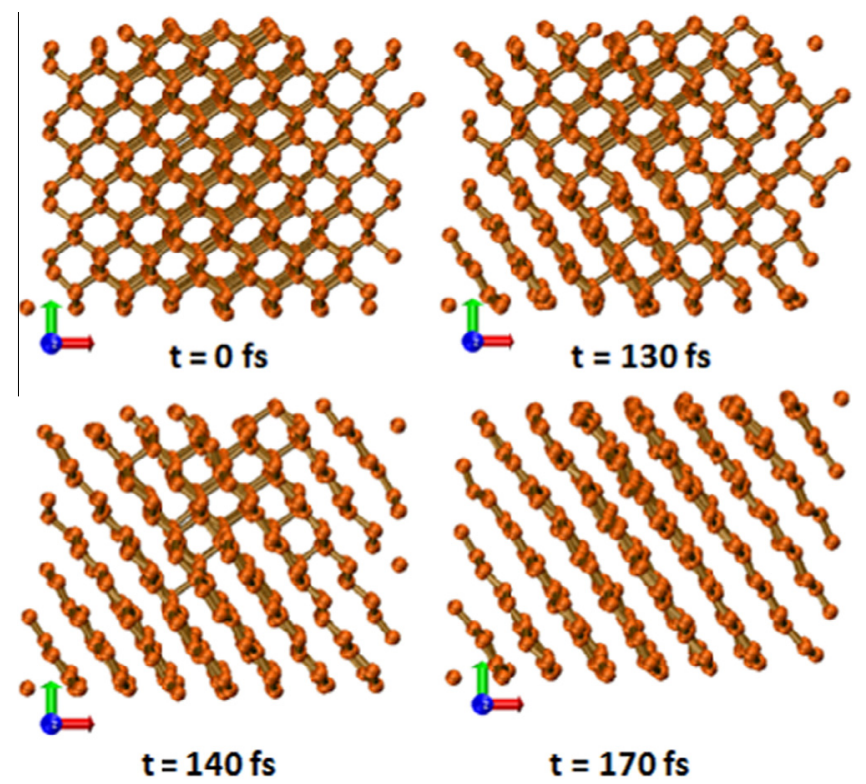

Fig. 8. Atomic snapshots of the nonthermal graphitization of diamond under FEL irradiation of $10 \mathrm{keV}$ photon energy, $10 \mathrm{fs}$ pulse, $0.85 \mathrm{eV} /$ atom absorbed dose. The figure is reproduced from Ref. [82].

\subsection{Examples: atomic dynamics after an SHI impact}

In contrast to the X-ray FEL-case, when a solid is irradiated with a swift-heavy ion, strong gradients of excitation in the electronic system occur, affecting considerably the relaxation kinetics. To account for them, we used MC code TREKIS described above, combined with other approaches to model a track formation. An inhouse hybrid code $^{3}$ couples TREKIS with another low-energyelectron kinetics tool (an approximate Boltzmann equation), and classical MD simulating the dynamics of excited lattice [83]. The

${ }^{3}$ Developed by S. Gorbunov, P. Terekhin, N. Medvedev, R. Rymzhanov, and A. Volkov, 2013.
DSF-formalism (Eq. (1)) is used to describe the electron-lattice coupling.

An example of the track kinetics in $\mathrm{Al}_{2} \mathrm{O}_{3}$ after Xe ion impact (167 MeV) was presented in [62]. It was shown that when electron coupling to the lattice was assumed within free-electron approximation for the conduction-band electrons appearing in a track (Eq. (1)), without contribution of valence-band holes, the resulting lattice heating seemed to be too low to produce experimentally observed damage regions, see Ref. [62]. Only taking into account the contribution from the excess energy of valence-band holes (in admittedly crude way), the track radius could be reproduced close to the experimental size [62]. The atomic snapshots are shown in Fig. 9 from the top-view. The importance of holes for the track kinetics seems to be coming from the fact that extremely fast electron transport out of the SHI trajectory carries energy away (as we saw in Fig. 3, and was discussed for a long time in the literature, see e.g. Refs. $[3,31,84-86])$. In contrast, valence holes are much slower (their kinetic energy is limited by the width of the valence band). Thus, these holes have enough time to interact with the atoms in the nanometric region around the ion trajectory providing them with energy sufficient to form a track.

Contribution of valence holes to the lattice heating is often neglected in theoretical studies of SHI track formation. This results into a need for adjusting the energy transfer to the lattice by artificially changing parameters of the electron-phonon coupling to heat the lattice more significantly in the center - before the electrons escape (see applications of the 'thermal-spike' model [60]). It might also be one of the reasons why a rescaling of input data from TTM for MD simulations is often performed [87,88]. The need for rescaling depends on interatomic potential used and the fact that softening of the potentials due to nonthermal effects is usually missing in classical-MD simulations. All of these effects and their relative importance warrant further dedicated research. Including the energy exchange between the lattice and valence holes seems to be necessary to avoid such fitting procedures. Another effect of valence holes that is often neglected is the Coulomb attraction of the excited electrons that might slow down their spreading. More detailed studies of these effects are currently in progress.

To the best of our knowledge, nonthermal melting in SHI tracks has not been studied quantitatively so far, although possibility of nonthermal melting is discussed in the literature [89-91]. Extreme levels of the initial electronic excitation, and their nanometric spatial localization resulting in strong spatial gradients inducing fast transport of particles and energy, pose challenges for theories, precluding direct application of previously developed schemes used for laser excitations. Moreover, when the spatial scales become smaller than the scales of the collective behavior of atoms (phonon wavelength), the two representations: real-space (coordinate), and reciprocal-space (momentum), are non-equivalent. That presents additional challenges for theoretical treatments of coupled electron and atomic dynamics in SHI tracks to be solved in future.

\section{Summary}

In this brief review we discussed similarities and differences in transient material excitation and evolution caused by freeelectron-laser and swift-heavy-ion irradiation. The starting points for these kinds of irradiation are obviously different: photoabsorption delivers quanta of energy, whereas an ion impact produces many electrons with different energies (initial spectrum approximately follows $\sim 1 / E^{2}$ ). Also, the spatial profile of an FEL beam creates different geometry compared with an ion impact. In spite of these differences in the initial spectra of the energy deposition, the basic mechanisms of the subsequent electron relaxation processes are essentially the same. Coupling to the lattice and further 
a

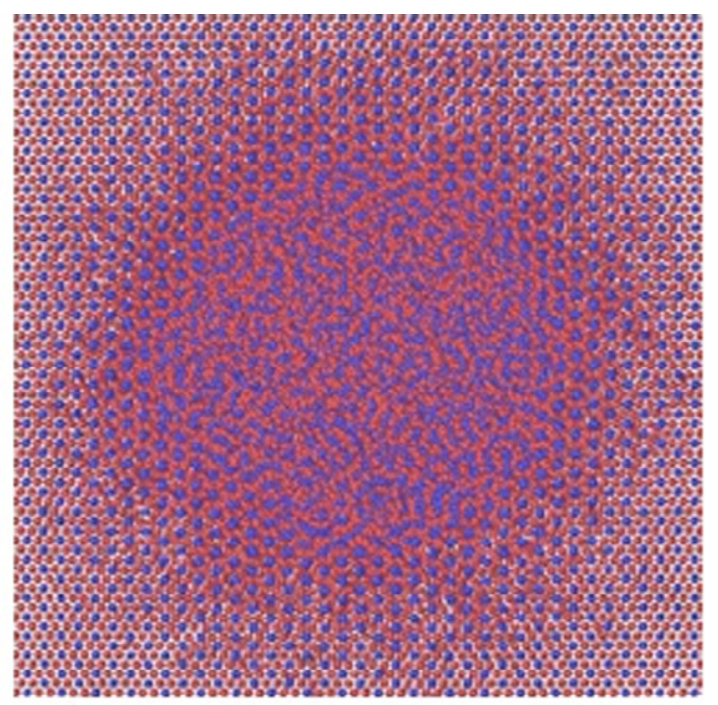

$\mathrm{b}$

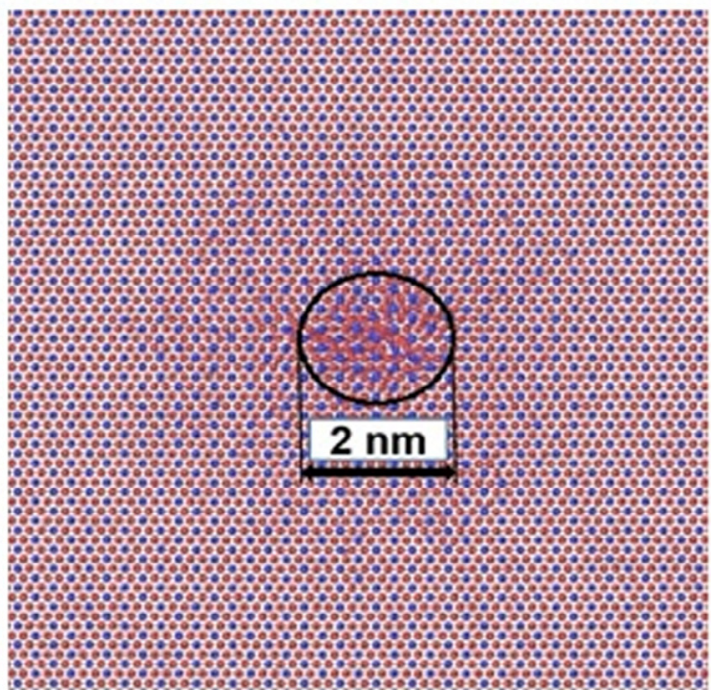

Fig. 9. Atomic snapshots of $\mathrm{Al}_{2} \mathrm{O}_{3}$ after Xe ion impact ( $167 \mathrm{MeV}$ ). (a) 1 ps after the ion passage; (b) $50 \mathrm{ps}$ after the ion passage. Al atoms are depicted as blue, $\mathrm{O}$ atoms are shown in red. Figure is reproduced from Ref. [62]. (For interpretation of the references to color in this figure legend, the reader is referred to the web version of this article.)

atomic dynamics also have the same underlying physics. The relative importance of different channels of energy dissipation is determined by the geometry and initial spectrum of excitation with FEL vs SHI irradiation.

Taking this into account, two methods of description of electron-lattice coupling appropriate for FEL and SHI irradiation are discussed: free-electron approximation and tight-binding approach. Based on these methods, the possibilities of thermal and nonthermal melting in FEL-irradiated spots were demonstrated; an insufficient heating of the lattice solely by excited free electrons in SHI tracks is discussed. For spatially inhomogeneous excitations, strong gradients of density and energy-density pose challenges for theoretical descriptions. They should be addressed in future modeling tools.

\section{Acknowledgements}

The authors thank S. Gorbunov, H. Jeschke, Z. Li, O. Osmani, B. Rethfeld, R. Rymzhanov, R. Santra, K. Schwartz, P. Terekhin, C. Trautmann, O. Vendrell for their contribution to the previous works which were discussed throughout this review, and for numerous valuable discussions. Partial financial support from Grants 13-02-1020, 15-02-02875, 15-58-15002 of Russian Foundation for Basic Research is acknowledged by A.E. Volkov.

\section{References}

[1] A. Ng, Outstanding questions in electron-ion energy relaxation, lattice stability, and dielectric function of warm dense matter, Int. J. Quantum Chem. 112 (2012) 150-160, http://dx.doi.org/10.1002/qua.23197.

[2] M.W.C. Dharma-wardana, F. Perrot, Energy relaxation and the quasiequation of state of a dense two-temperature nonequilibrium plasma, Phys. Rev. E 58 (1998) 3705-3718, http://dx.doi.org/10.1103/PhysRevE.58.3705.

[3] A.E. Volkov, V.A. Borodin, The rate of the electron-ion heat transfer in swift heavy particle tracks in metals, Nucl. Instr. Meth. Phys. Res. Sect. B Beam Interact. Mater. Atoms 107 (1996) 172-174, http://dx.doi.org/10.1016/0168583X(95)01031-9.

[4] A. Kaiser, B. Rethfeld, M. Vicanek, G. Simon, Microscopic processes in dielectrics under irradiation by subpicosecond laser pulses, Phys. Rev. B 61 (2000) 11437-11450, http://dx.doi.org/10.1103/PhysRevB.61.11437.

[5] T.G. White, J. Vorberger, C.R.D. Brown, B.J.B. Crowley, P. Davis, S.H. Glenzer et al., Observation of inhibited electron-ion coupling in strongly heated graphite, Sci. Rep. 2 (2012) 889, http://dx.doi.org/10.1038/srep00889.

[6] P. Lorazo, L. Lewis, M. Meunier, Thermodynamic pathways to melting, ablation, and solidification in absorbing solids under pulsed laser irradiation, Phys. Rev. B 73 (2006) 134108, http://dx.doi.org/10.1103/PhysRevB.73.134108.

[7] D.R. Rittman, C.L. Tracy, A.B. Cusick, M.J. Abere, B. Torralva, R.C. Ewing, et al., Ultrafast laser and swift heavy ion irradiation: response of $\mathrm{Gd}_{2} \mathrm{O}_{3}$ and $\mathrm{ZrO}_{2}$ to intense electronic excitation, Appl. Phys. Lett. 106 (2015) 171914, http://dx. doi.org/10.1063/1.4919720.

[8] B. Rethfeld, A. Kaiser, M. Vicanek, G. Simon, Ultrafast dynamics of nonequilibrium electrons in metals under femtosecond laser irradiation, Phys. Rev. B 65 (2002) 214303, http://dx.doi.org/10.1103/ PhysRevB.65.214303.

[9] V.P. Lipp, A.E. Volkov, M.V. Sorokin, B. Rethfeld, Kinetics of propagation of the lattice excitation in a swift heavy ion track, Nucl. Instr. Meth. Phys. Res. Sect. B Beam Interact. Mater. Atoms 269 (2011) 865-868, http://dx.doi.org/10.1016/j. nimb.2010.11.070.

[10] R.R. Fäustlin, T. Bornath, T. Döppner, S. Düsterer, E. Förster, C. Fortmann, et al., Observation of ultrafast nonequilibrium collective dynamics in warm dense hydrogen, Phys. Rev. Lett. 104 (2010) 125002, http://dx.doi.org/10.1103/ PhysRevLett. 104.125002.

[11] N. Medvedev, U. Zastrau, E. Förster, D.O. Gericke, B. Rethfeld, Short-time electron dynamics in aluminum excited by femtosecond extreme ultraviolet radiation, Phys. Rev. Lett. 107 (2011) 165003, http://dx.doi.org/10.1103/ PhysRevLett. 107.165003.

[12] B. Rethfeld, A. Rämer, N. Brouwer, N. Medvedev, O. Osmani, Electron dynamics and energy dissipation in highly excited dielectrics, Nucl. Instr. Meth. Phys. Res. B. 327 (2014) 78-88, http://dx.doi.org/10.1016/j.nimb.2013.10.087.

[13] W. Ackermann, G. Asova, V. Ayvazyan, A. Azima, N. Baboi, J. Bähr, et al., Operation of a free-electron laser from the extreme ultraviolet to the water window, Nat. Photonics 1 (2007) 336-342, http://dx.doi.org/10.1038/ nphoton.2007.76.

[14] P. Emma, R. Akre, J. Arthur, R. Bionta, C. Bostedt, J. Bozek, et al., First lasing and operation of an ångstrom-wavelength free-electron laser, Nat. Photonics 4 (2010) 641-647, http://dx.doi.org/10.1038/nphoton.2010.176.

[15] D. Pile, X-rays: first light from SACLA, Nat. Photonics 5 (2011) 456-457, http:// dx.doi.org/10.1038/nphoton.2011.178.

[16] E. Allaria, R. Appio, L. Badano, W.A. Barletta, S. Bassanese, S.G. Biedron, et al. Highly coherent and stable pulses from the FERMI seeded free-electron laser in the extreme ultraviolet, Nat. Photonics 6 (2012) 699-704, http://dx.doi.org/ 10.1038/nphoton.2012.233.

[17] M. Nakatsutsumi, K. Appel, G. Priebe, I. Thorpe, A. Pelka, B. Muller, et al. Technical design report: scientific instrument, high energy density physics (HED), scientific, European X-Ray Free-Electron Laser Facility GmbH, Hamburg, Germany, 2014. <https://docs.xfel.eu/alfresco/d/a/workspace/ SpacesStore/7ce26dad-b8df-42>.

[18] B.L. Henke, E.M. Gullikson, J.C. Davis, X-Ray interactions: photoabsorption, scattering, transmission, and reflection at $E=50-30,000 \mathrm{eV}, Z=1-92$, At. Data Nucl. Data Tables 54 (1993) 181-342, http://dx.doi.org/10.1006/ adnd.1993.1013.

[19] E.D. Palik, Handbook of Optical Constants of Solids, Academic Press, 1985, http://dx.doi.org/10.1016/B978-012544415-6.50002-9.

[20] K. Schwartz, A.E. Volkov, K.-O. Voss, M.V. Sorokin, C. Trautmann, R. Neumann, Thermal spike effect on defect evolution in $\mathrm{NaCl}$ irradiated with light and heavy ions at 8 and $300 \mathrm{~K}$, Nucl. Instr. Meth. Phys. Res. Sect. B Beam Interact. Mater. Atoms 245 (2006) 204-209, http://dx.doi.org/10.1016/j. nimb.2005.11.102.

[21] N.A. Medvedev, A.E. Volkov, N.S. Shcheblanov, B. Rethfeld, Early stage of the electron kinetics in swift heavy ion tracks in dielectrics, Phys. Rev. B 82 (2010) 125425, http://dx.doi.org/10.1103/PhysRevB.82.125425.

[22] J.F. Littmark, J.P. Ziegler, U. Biersack, The Stopping and Range of Ions in Solids, Pergamon, New York, 1985. <http://www.srim.org>. 
[23] D. Fink (Ed.), Fundamentals of Ion-Irradiated Polymers, Springer, Berlin Heidelberg, Berlin, 2004.

[24] F. Aumayr, S. Facsko, A.S. El-Said, C. Trautmann, M. Schleberger, Single ion induced surface nanostructures: a comparison between slow highly charged and swift heavy ions, J. Phys. Condens. Matter 23 (2011) 393001, http://dx.doi. org/10.1088/0953-8984/23/39/393001.

[25] K. Schwartz, A. Volkov, M. Sorokin, C. Trautmann, K.-O. Voss, R. Neumann, et al., Effect of electronic energy loss and irradiation temperature on colorcenter creation in $\mathrm{LiF}$ and $\mathrm{NaCl}$ crystals irradiated with swift heavy ions, Phys. Rev. B 78 (2008) 024120, http://dx.doi.org/10.1103/PhysRevB.78.024120.

[26] M. Harmand, R. Coffee, M. Bionta, M. Chollet, D. French, D.M. Zhu, et al., Achieving few-femtosecond time-sorting at hard X-ray free-electron lasers, Nat. Photonics 7 (2013) 215-218, http://dx.doi.org/10.1038/nphoton.2013.11.

[27] R. Sobierajski, M. Jurek, J. Chalupský, J. Krzywinski, T. Burian, S.D. Farahani, et al., Experimental set-up and procedures for the investigation of XUV free electron laser interactions with solids, J. Instr. 8 (2013) P02010, http://dx.doi. org/10.1088/1748-0221/8/02/P02010.

[28] S.-K. Son, L. Young, R. Santra, Impact of hollow-atom formation on coherent $\mathrm{x}$ ray scattering at high intensity, Phys. Rev. A 83 (2011) 033402, http://dx.doi. org/10.1103/PhysRevA.83.033402.

[29] A.A. Baranov, N.A. Medvedev, A.E. Volkov, N.S. Scheblanov, Effect of interaction of atomic electrons on ionization of an insulator in swift heavy ion tracks, Nucl. Instr. Meth. Phys. Res. Sect. B Beam Interact. Mater. Atoms 286 (2012) 51-55, http://dx.doi.org/10.1016/j.nimb.2012.01.011.

[30] N. Medvedev, Z. Li, B. Ziaja, Thermal and nonthermal melting of silicon under femtosecond x-ray irradiation, Phys. Rev. B 91 (2015) 054113, http://dx.doi. org/10.1103/PhysRevB.91.054113.

[31] A.E. Volkov, V.A. Borodin, Heating of metals in swift heavy ion tracks by electron-ion energy exchange, Nucl. Instr. Meth. Phys. Res. Sect. B Beam Interact. Mater. Atoms 146 (1998) 137-141, http://dx.doi.org/10.1016/S0168583X(98)00454-6.

[32] B. Gervais, S. Bouffard, Simulation of the primary stage of the interaction of swift heavy ions with condensed matter, Nucl. Instr. Meth. Phys. Res. Sect. B Beam Interact. Mater. Atoms 88 (1994) 355-364, http://dx.doi.org/10.1016 0168-583X(94)95384-8.

[33] E. Kobetich, R. Katz, Energy deposition by electron beams and $\delta$ rays, Phys. Rev. 170 (1968) 391-396, http://dx.doi.org/10.1103/PhysRev. 170.391.

[34] M.P.R. Waligórski, R.N. Hamm, R. Katz, O. Ridge, The radial distribution of dose around the path of a heavy ion in liquid water, Int. J. Radiat. Appl. Instr. Part D. Nucl. Tracks Radiat. Meas. 11 (1986) 309-319, http://dx.doi.org/10.1016/ 1359-0189(86)90057-9.

[35] D.D. Ryutov, Multipulse effects in the damage to the LCLS reflective optics, in: S.G. Biedron, W. Eberhardt, T. Ishikawa, R.O. Tatchyn (Eds.), Opt. Sci. Technol. SPIE 49th Annu. Meet., International Society for Optics and Photonics, 2004 pp. 58-65. http://dx.doi.org/10.1117/12.559091.

[36] N. Itoh, D.M. Duffy, S. Khakshouri, A.M. Stoneham, Making tracks: electronic excitation roles in forming swift heavy ion tracks, J. Phys. Condens. Matter 21 (2009) 474205, http://dx.doi.org/10.1088/0953-8984/21/47/474205.

[37] M.R. Islam, U. Saalmann, J.M. Rost, Kinetic energy of ions after Coulomb explosion of clusters induced by an intense laser pulse, Phys. Rev. A 73 (2006) 041201, http://dx.doi.org/10.1103/PhysRevA.73.041201.

[38] B. Ziaja, H. Wabnitz, F. Wang, E. Weckert, T. Möller, Energetics, ionization, and expansion dynamics of atomic clusters irradiated with short intense vacuumultraviolet pulses, Phys. Rev. Lett. 102 (2009) 205002, http://dx.doi.org/ 10.1103/PhysRevLett. 102.205002.

[39] P.B. Allen, Theory of thermal relaxation of electrons in metals, Phys. Rev. Lett. 59 (1987) 1460-1463, http://dx.doi.org/10.1103/PhysRevLett. 59.1460.

[40] L.D. Pietanza, G. Colonna, S. Longo, M. Capitelli, Non-equilibrium electron and phonon dynamics in metals under femtosecond laser pulses, Eur. Phys. J. D 45 (2007) 369-389, http://dx.doi.org/10.1140/epjd/e2007-00251-1.

[41] N.S. Shcheblanov, T.E. Itina, Femtosecond laser interactions with dielectric materials: insights of a detailed modeling of electronic excitation and relaxation processes, Appl. Phys. A 110 (2012) 579-583, http://dx.doi.org/ 10.1007/s00339-012-7130-0.

[42] B.Y. Mueller, B. Rethfeld, Relaxation dynamics in laser-excited metals under nonequilibrium conditions, Phys. Rev. B 87 (2013) 035139, http://dx.doi.org/ 10.1103/PhysRevB.87.035139.

[43] C. Jacoboni, L. Reggiani, The Monte Carlo method for the solution of charge transport in semiconductors with applications to covalent materials, Rev. Mod. Phys. 55 (1983) 645-705, http://dx.doi.org/10.1103/RevModPhys. 55.645.

[44] T. Boutboul, A. Akkerman, A. Breskin, R. Chechik, Electron inelastic mean free path and stopping power modelling in alkali halides in the $50 \mathrm{eV}-10 \mathrm{keV}$ energy range, J. Appl. Phys. 79 (1996) 6714, http://dx.doi.org/10.1063/1.361491.

[45] N. Medvedev, B. Rethfeld, Transient dynamics of the electronic subsystem of semiconductors irradiated with an ultrashort vacuum ultraviolet laser pulse, New J. Phys. 12 (2010) 073037, http://dx.doi.org/10.1088/1367-2630/12/7/ 073037.

[46] N. Medvedev, Femtosecond X-ray induced electron kinetics in dielectrics: application for FEL-pulse-duration monitor, Appl. Phys. B 118 (2015) 417-429, http://dx.doi.org/10.1007/s00340-015-6005-4.

[47] N. Medvedev, B. Ziaja, M. Cammarata, M. Harmand, S. Toleikis, Electron kinetics in femtosecond X-ray irradiated $\mathrm{SiO}_{2}$, Contrib. Plasma Phys. 53 (2013) 347-354, http://dx.doi.org/10.1002/ctpp.201200095.

[48] O. Keski-Rahkonen, M.O. Krause, Total and partial atomic-level widths, At. Data Nucl. Data Tables 14 (1974) 139-146, http://dx.doi.org/10.1016/S0092 640X(74)80020-3.
[49] S.P. Hau-Riege, Nonequilibrium electron dynamics in materials driven by highintensity x-ray pulses, Phys. Rev. E 87 (2013) 053102, http://dx.doi.org/ 10.1103/PhysRevE.87.053102.

[50] N. Medvedev, H.O. Jeschke, B. Ziaja, Nonthermal graphitization of diamond induced by a femtosecond x-ray laser pulse, Phys. Rev. B 88 (2013) 224304, http://dx.doi.org/10.1103/PhysRevB.88.224304.

[51] D.A. Chapman, D.O. Gericke, Analysis of Thomson scattering from nonequilibrium plasmas, Phys. Rev. Lett. 107 (2011) 165004, http://dx.doi. org/10.1103/PhysRevLett. 107.165004.

[52] S.M. Vinko, U. Zastrau, S. Mazevet, J. Andreasson, S. Bajt, T. Burian, et al., Electronic structure of an XUV photogenerated solid-density aluminum plasma, Phys. Rev. Lett. 104 (2010) 225001, http://dx.doi.org/10.1103/ PhysRevLett. 104.225001.

[53] U. Zastrau, C. Fortmann, R. Fäustlin, L. Cao, T. Döppner, S. Düsterer, et al., Bremsstrahlung and line spectroscopy of warm dense aluminum plasma heated by xuv free-electron-laser radiation, Phys. Rev. E 78 (2008) 066406 , http://dx.doi.org/10.1103/PhysRevE.78.066406.

[54] N.A. Medvedev, R.A. Rymzhanov, A.E. Volkov, Time resolved electron kinetics in swift-heavy ion irradiated solids, J. Phys. D: Appl. Phys. 48 (2015), http://dx. doi.org/10.1088/0022-3727/48/35/355303. 355303 (24 pp.).

[55] R.A. Rymzhanov, N.A. Medvedev, A.E. Volkov, Electron emission from silicon and germanium after swift heavy ion impact, Phys. Status Solidi 252 (2015) 159-164, http://dx.doi.org/10.1002/pssb.201400130.

[56] A. Akkerman, M. Murat, J. Barak, Delta-electron spectra, inelastic cross sections, and stopping powers of ions in silicon: comparison between different models, Nucl. Instr. Meth. Phys. Res. Sect. B Beam Interact. Mater. Atoms 321 (2014) 1-7, http://dx.doi.org/10.1016/j.nimb.2013.12.002.

[57] G. Schiwietz, K. Czerski, M. Roth, F. Staufenbiel, P.L. Grande, Femtosecond dynamics - snapshots of the early ion-track evolution, Nucl. Instr. Meth. Phys. Res. Sect. B Beam Interact. Mater. Atoms 226 (2004) 683-704, http://dx.doi. org/10.1016/j.nimb.2004.05.043.

[58] W. Eckstein, Computer Simulation of Ion-Solid Interactions, Springer, Berlin Heidelberg, 2011. https://books.google.com/books?id=rzVtMAEACAAJ\&pgis=1 (accessed April 11, 2015)

[59] O. Osmani, N. Medvedev, M. Schleberger, B. Rethfeld, Energy dissipation in dielectrics after swift heavy-ion impact: a hybrid model, Phys. Rev. B 84 (2011) 214105, http://dx.doi.org/10.1103/PhysRevB.84.214105.

[60] M. Toulemonde, C. Dufour, E. Paumier, Transient thermal process after a highenergy heavy-ion irradiation of amorphous metals and semiconductors, Phys. Rev. B 46 (1992) 14362-14369, http://dx.doi.org/10.1103/PhysRevB.46.14362.

[61] M. Murat, A. Akkerman, J. Barak, Ion track structure and dynamics in $\mathrm{SiO}_{2}$, in: 2007 9th Eur. Conf. Radiat. its Eff. Components Syst., vol. 55, 2008, pp. 21132120. http://dx.doi.org/10.1109/RADECS.2007.5205478.

[62] P.N. Terekhin, R.A. Rymzhanov, S.A. Gorbunov, N.A. Medvedev, A.E. Volkov, Effect of valence holes on swift heavy ion track formation in $\mathrm{Al}_{2} \mathrm{O}_{3}$, Nucl. Instr. Meth. Phys. Res. Sect. B Beam Interact. Mater. Atoms 354 (2015) 200-204, http://dx.doi.org/10.1016/j.nimb.2015.01.069.

[63] N.W. Ashcroft, D.N. Mermin, Solid State Physics, first ed., Cengage Learning, Boston, 1976

[64] P. Stampfli, K. Bennemann, Theory for the instability of the diamond structure of $\mathrm{Si}, \mathrm{Ge}$, and $\mathrm{C}$ induced by a dense electron-hole plasma, Phys. Rev. B 42 (1990) 7163-7173, http://dx.doi.org/10.1103/PhysRevB.42.7163.

[65] P. Stampfli, K. Bennemann, Dynamical theory of the laser-induced lattice instability of silicon, Phys. Rev. B 46 (1992) 10686-10692, http://dx.doi.org/ 10.1103/PhysRevB.46.10686.

[66] L. Van Hove, Correlations in space and time and born approximation scattering in systems of interacting particles, Phys. Rev. 95 (1954) 249-262, http://dx.doi. org/10.1103/PhysRev. 95.249.

[67] G. Baym, Direct calculation of electronic properties of metals from neutron scattering data, Phys. Rev. 135 (1964) A1691-A1692, http://dx.doi.org/ 10.1103/PhysRev. 135.A1691.

[68] J. Chihara, Difference in X-ray scattering between metallic and non-metallic liquids due to conduction electrons, J. Phys. F Met. Phys. 17 (1987) 295-304, http://dx.doi.org/10.1088/0305-4608/17/2/002.

[69] J. Chihara, Interaction of photons with plasmas and liquid metals photoabsorption and scattering, J. Phys. Condens. Matter 12 (2000) 231-247, http://dx.doi.org/10.1088/0953-8984/12/3/303.

[70] R.W. Lee, S.J. Moon, H.-K. Chung, W. Rozmus, H.A. Baldis, G. Gregori, et al., Finite temperature dense matter studies on next-generation light sources, J. Opt. Soc. Am. B 20 (2003) 770, http://dx.doi.org/10.1364/JOSAB.20.000770.

[71] M.P. Tosi, M. Parrinello, N.H. March, Electronic effects in dynamical structure of liquid metals, Nuovo Cim. B 23 (1974) 135-171, http://dx.doi.org/10.1007/ BF02737503.

[72] N.H. March, M.P. Tosi, Atomic Dynamics in Liquids, Courier Corporation, Chelmsford, 1991. https://books.google.com/books?id=B7gZWY5m6fgC\&pgis= 1 (accessed April 10, 2015)

[73] V.T. Shvets, A.P. Fedtchuk, Electron-phonon interaction and electronic transport phenomena in amorphous transition metals, Phys. Scr. 52 (1995) 722-725, http://dx.doi.org/10.1088/0031-8949/52/6/021.

[74] S.A. Gorbunov, N.A. Medvedev, P.N. Terekhin, A.E. Volkov, Electron-lattice coupling after high-energy deposition in aluminum, Nucl. Instr. Meth. Phys. Res. Sect. B Beam Interact. Mater. Atoms 354 (2014) 220-225, http://dx.doi. org/10.1016/j.nimb.2014.11.053.

[75] J.-C. Kuhr, H.-J. Fitting, Monte Carlo simulation of electron emission from solids, J. Electron Spectrosc. Relat. Phenom. 105 (1999) 257-273, http://dx.doi. org/10.1016/S0368-2048(99)00082-1. 
[76] R. Kubo, The fluctuation-dissipation theorem, Rep. Prog. Phys. 29 (1966) 255284, http://dx.doi.org/10.1088/0034-4885/29/1/306.

[77] H. Jeschke, M. Garcia, K. Bennemann, Microscopic analysis of the laser-induced femtosecond graphitization of diamond, Phys. Rev. B 60 (1999) R3701-R3704, http://dx.doi.org/10.1103/PhysRevB.60.R3701.

[78] N. Medvedev, H.O. Jeschke, B. Ziaja, Nonthermal phase transitions in semiconductors induced by a femtosecond extreme ultraviolet laser pulse, New J. Phys. 15 (2013) 015016, http://dx.doi.org/10.1088/1367-2630/15/1/ 015016.

[79] J. Gaudin, N. Medvedev, J. Chalupský, T. Burian, S. Dastjani-Farahani, V. Hájková, et al., Photon energy dependence of graphitization threshold for diamond irradiated with an intense XUV FEL pulse, Phys. Rev. B 88 (2013) 060101(R), http://dx.doi.org/10.1103/PhysRevB.88.060101.

[80] M. Beye, F. Sorgenfrei, W.F. Schlotter, W. Wurth, A. Föhlisch, The liquid-liquid phase transition in silicon revealed by snapshots of valence electrons, Proc. Natl. Acad. Sci. USA 107 (2010) 16772-16776, http://dx.doi.org/10.1073/ pnas.1006499107.

[81] E. Zijlstra, A. Kalitsov, T. Zier, M. Garcia, Squeezed thermal phonons precurse nonthermal melting of silicon as a function of fluence, Phys. Rev. X 3 (2013) 011005, http://dx.doi.org/10.1103/PhysRevX.3.011005.

[82] N. Medvedev, V. Tkachenko, B. Ziaja, Modeling of nonthermal solid-to-solid phase transition in diamond irradiated with femtosecond x-ray FEL pulse, Contrib. Plasma Phys. 55 (2015) 12-34, http://dx.doi.org/10.1002/ ctpp.201400026.

[83] S.A. Gorbunov, N.A. Medvedev, R.A. Rymzhanov, P.N. Terekhin, A.E. Volkov, Excitation and relaxation of olivine after swift heavy ion impact, Nucl. Instr.
Meth. Phys. Res. B 326 (2014) 163-168, http://dx.doi.org/10.1016/j. nimb.2013.09.028.

[84] M.I. Kaganov, I.M. Lifshitz, L.V. Tanatarov, Relaxation between electrons and crystalline lattice, Sov. Phys. JETP 4 (1957) 173.

[85] I.A. Baranov, Y.V. Martynenko, S.O. Tsepelevich, Y.N. Yavlinskii, Inelastic sputtering of solids by ions, Phys.-Usp. 31 (1988) 1015-1034. http://ufn.ru/en/ articles/1988/11/c/ (accessed June 27, 2013).

[86] Y.V. Martynenko, Y.N. Yavlinskii, Fission-fragment excitation of metal electrons, Sov. At. Energy 62 (1987) 93-97, http://dx.doi.org/10.1007/ BF01123661.

[87] M.C. Ridgway, T. Bierschenk, R. Giulian, B. Afra, M.D. Rodriguez, L.L. Araujo, et al., Tracks and voids in amorphous Ge induced by swift heavy-ion irradiation, Phys. Rev. Lett. 110 (2013) 245502, http://dx.doi.org/10.1103/ PhysRevLett. 110.245502.

[88] T. Bierschenk, R. Giulian, B. Afra, M.D. Rodriguez, D. Schauries, S. Mudie, et al. Latent ion tracks in amorphous silicon, Phys. Rev. B 88 (2013) 174111, http:// dx.doi.org/10.1103/PhysRevB.88.174111.

[89] D.M. Duffy, S.L. Daraszewicz, J. Mulroue, Modelling the effects of electronic excitations in ionic-covalent materials, Nucl. Instr. Meth. Phys. Res. Sect. B Beam Interact. Mater. Atoms 277 (2012) 21-27, http://dx.doi.org/10.1016/j. nimb.2011.12.059.

[90] V.V. Stegailov, Stability of LiF crystal in the warm dense matter state, Contrib. Plasma Phys. 50 (2010) 31-34, http://dx.doi.org/10.1002/ctpp.201010008.

[91] M. Murat, A. Akkerman, J. Barak, Can swift heavy ions create latent tracks in silicon?, Nucl Instr. Meth. Phys. Res. Sect. B Beam Interact. Mater. Atoms 269 (2011) 2649-2656, http://dx.doi.org/10.1016/j.nimb.2011.07.097. 